\title{
SELECTED IMMUNOHISTOCHEMICAL FEATURES OF CONVENTIONAL RENAL CELL CARCINOMAS COEXPRESSING P53 AND MDM2
}

\author{
Maria Hejnold $^{1}$, Grzegorz Dyduch ${ }^{1}$, Magdalena Bialas ${ }^{1}$, Sergiusz Demczuk ${ }^{1}$, Janusz Ryś ${ }^{3}$, \\ Piotr ChŁosta ${ }^{2}$, Tomasz Szopiński ${ }^{2}$, Krzysztof Okoń $^{1}$
}

\author{
${ }^{1}$ Department of Pathomorphology, Collegium Medicum, Jagiellonian University, Krakow, Poland \\ ${ }^{2}$ Department of Urology, Collegium Medicum, Jagiellonian University, Krakow, Poland \\ ${ }^{3}$ Department of Pathology, Centre of Oncology, Krakow, Poland
}

\begin{abstract}
Renal clear cell carcinoma (CCRCC) is an aggressive tumor for which new prognostic factors are needed. It has been suggested that CCRCCs co-expressing P53 and MDM2 could represent a special subgroup; therefore the aim of this study was to explore their immunohistochemical features. The material studied consisted of 470 cases of CCRCC. Immunohistochemistry for MDM2, P53, Ki-67, VEGF-A, VEGF-C, VEGF-D, GLUT1, CA9, and CK 7 was performed on tissue microarrays and assessed semi-quantitatively. On average, $6.6 \%$ or $5.3 \%$ of cases were $\mathrm{P} 53+/$ MDM2+, depending on the P53 antibody used. The mean percentage of $\mathrm{Ki}-67$ positive cells was $0.6 \%$ and p 53 -positive MDM2-positive cases showed significantly higher expression of Ki-67. The other immunohistochemical parameters studied did not differ between p53-positive MDM2-positive cases and the rest of the subtypes studied. Expression of almost all immunohistochemical markers differed with respect to $\mathrm{pT}$ stage; only for CA9 was the difference not significant. Furthermore, almost all immunohistochemical markers studied differed with respect to differences in grade; only for GLUT1 was the difference not significant. Our results suggest that with the exception of $\mathrm{Ki}-67$, there are no significant associations between analyzed markers and the double P53+/MDM2+ phenotype.
\end{abstract}

Key words: renal cell carcinoma, MDM2, P53, Ki-67, VEGF-A, VEGF-C, VEGF-D, GLUT1, CK 7, CA9.

\section{Introduction}

Renal cell carcinoma (RCC) constitutes approximately $9 \%$ of all human cancers. The most frequent variant, conventional or clear cell (CCRCC), is particularly interesting because of the considerable progress obtained in the last few years and the introduction of targeted therapy. In CCRCC patients, the prognosis is dependent on stage, grade and patient clinical condition. Other prognostic markers, including immunohistochemistry, have been studied; however, none have been introduced into clinical practice. It has been suggested that co-expression of P53 and
MDM2 may define a distinct group of CCRCCs [1]. In our previous work [2] we explored this hypothesis by comparing the histologic prognostic factors such as stage, grade or presence of necrosis in tumors with or without doubly-expressed P53 and MDM2. The aim of the current study is to analyze the expression of potential immunohistochemical markers including Ki-67, VEGF-A, VEGF-C, VEGF-D, GLUT1, CK 7 and CA9 in samples expressing both P53 and MDM2, and to determine whether these immunohistochemical markers have prognostic significance. 
Table I. Antibodies used in this study

\begin{tabular}{lccc}
\hline SPECIFICITY & Dilution & MANUfACtURER & CLONE/TYPE \\
\hline MDM2 & $1: 50$ & Novocastra & 1 B10 \\
\hline P53 & $1: 200$ & DAKO & DO-7 \\
\hline P53 & $1: 50$ & Novocastra & PAb1801 \\
\hline Ki-67 & $1: 50$ & DAKO & MIB-1 \\
\hline VEGF-A & $1: 100$ & Santa Cruz & polyclonal A20 \\
\hline VEGF-C & $1: 100$ & Santa Cruz & polyclonal H-190 \\
\hline VEGF-D & $1: 200$ & R\&D systems & 78923 \\
\hline GLUT1 & $1: 50$ & DAKO & polyclonal MYM \\
\hline CK 7 & $1: 50$ & DAKO & OV-TL 12/30 \\
\hline CA9 & $1: 100$ & Novocastra & TH22
\end{tabular}

\section{Material and methods}

The material analyzed was retrieved from the archives of the Department of Pathomorphology. Cases were reviewed by an expert urologic pathologist and reclassified according to the most recent WHO classification [3]. For the present study, only unequivocal conventional (clear cell) carcinomas were chosen. The tumors were graded according to the International Society of Urological Pathologists, which is derived from a modification of the Fuhrman method [4]; this is referred to as the Fuhrman method from this point onward. The presence of sarcomatoid components and necrosis was noticed, and in accordance with Delahunt et al. [5], another tumor grade was assigned that took necrosis into consideration.

For each case, a slide containing well preserved and representative tumor tissue was selected and a respective area was marked for study. Corresponding blocks were used to construct a tissue microarray (TMA) using Tissue MicroArrayer MTA-1 (Beecher Instruments Inc., Sun Prairie, USA). From each donor block, three $0.6 \mathrm{~mm}$ cylinders were selected. The acceptor paraffin blocks were prepared noting the location of each cylinder, and $3 \mu \mathrm{m}$-thick sections were cut.

Table II. Immunohistochemistry results

\begin{tabular}{lcccc}
\hline & Mean & Median & RANGe & SD \\
\hline VEGF-A & 1.12 & 1.00 & $0-3.00$ & 0.82 \\
\hline VEGF-C & 0.76 & 0.67 & $0-2.67$ & 0.66 \\
\hline VEGF-D & 0.30 & 0.00 & $0-3.00$ & 0.48 \\
\hline GLUT1 & 0.41 & 0.17 & $0-2.33$ & 0.53 \\
\hline CK7 & 0.16 & 0.00 & $0-1.00$ & 0.33 \\
\hline CA9 & 1.88 & 2.00 & $0-3.00$ & 0.96 \\
\hline
\end{tabular}

For immunohistochemistry, a standard staining protocol was used. Briefly, the slides were dewaxed, rehydrated and incubated in 3\% peroxide solution for 10 minutes to block endogenous peroxidase activity. Antigen retrieval was carried out by microwaving in citrate buffer $(0.2 \%$ citric acid titrated to $\mathrm{pH}$ 6.0 with $2 \mathrm{~N} \mathrm{NaOH}) 3$ times for 5 minutes each at $750 \mathrm{~W}$. The primary antibodies are listed in Table I. The Lab Vision detection system (Thermo Fisher Scientific, Waltham, USA) was used. The chromogen used was 3-amino-9-ethylcarbazole. The slides were counterstained with Mayer hematoxylin (Thermo Fisher Scientific, Waltham, USA) and coverslipped. The immunohistochemistry was scored by one of the authors (M.H.) without knowledge of the clinicopathologic parameters and the results of scoring introduced into an Excel spreadsheet (Microsoft Corp., Redmond, USA). Samples were classified as positive if they expressed both P53 and MDM2, and if not, they were marked as negative. Ki-67 expression was quantified based on the percentage of immunopositive cells. Furthermore, to determine expression of each of CA9, CK 7, CD10, VEGF-A, VEGF-C, and VEGF-D, a scale from 0 to 3 was used, where 0 represented no staining and 3 represented strong staining of all cells. The results were averaged across all the scores available for the given case.

Cases lost from the TMAs were excluded from the study. Student's t, $\chi^{2}$ and ANOVA tests were used where appropriate. Correlations were measured by Pearson's and gamma correlation coefficients. The statistical analysis was done with Statistica $10 \mathrm{PL}$ (StatSoft Inc., Tulsa, USA) and $\mathrm{P}$ values less than 0.05 were considered significant.

\section{Results}

The material studied was obtained from 470 cases. There were $280(59.6 \%)$ males and $190(40.4 \%)$ females. The mean age of the patients was 61.3 years 
Table III. Relationship between the immunohistochemistry results and pT stage

\begin{tabular}{|c|c|c|c|c|c|c|c|c|c|c|c|c|}
\hline & \multicolumn{2}{|c|}{ VEGF-A } & \multicolumn{2}{|c|}{ VEGF-C } & \multicolumn{2}{|c|}{ VEGF-D } & \multicolumn{2}{|c|}{ GLUT1 } & \multicolumn{2}{|c|}{ CK-7 } & \multicolumn{2}{|c|}{ CA9 } \\
\hline & MEAN & SD & MEAN & SD & MEAN & SD & MEAN & SD & MEAN & $\mathrm{SD}$ & MEAN & SD \\
\hline pT1 & 1.00 & 0.79 & 0.69 & 0.60 & 0.22 & 0.40 & 0.32 & 0.45 & 0.23 & 0.39 & 2.00 & 0.95 \\
\hline pT2 & 1.17 & 0.87 & 1.01 & 0.76 & 0.45 & 0.68 & 0.38 & 0.46 & 0.18 & 0.31 & 1.98 & 1.01 \\
\hline pT3 & 1.23 & 0.83 & 0.78 & 0.69 & 0.35 & 0.50 & 0.50 & 0.59 & 0.10 & 0.27 & 1.78 & 0.95 \\
\hline pT4 & 0.67 & 0.88 & 0.44 & 0.19 & 0.00 & 0.00 & 0.00 & 0.00 & 0.00 & 0.00 & 1.00 & 1.00 \\
\hline $\mathrm{p}$ & \multicolumn{2}{|c|}{$<0.03$} & \multicolumn{2}{|c|}{$<0.04$} & \multicolumn{2}{|c|}{$<0.01$} & \multicolumn{2}{|c|}{$<0.002$} & \multicolumn{2}{|c|}{$<0.002$} & \multicolumn{2}{|c|}{ NS } \\
\hline
\end{tabular}

(range 26 to 92; SD 10.59). P53 expression, when staining with the PAb1081 antibody, was positive in 62 cases $(13.2 \%)$. Furthermore, when using the p53-specific antibody, DO-7, expression was observed in 71 cases $(15.1 \%)$. Reaction for MDM2 was positive in 178 cases $(37.9 \%)$. There were 31 cases (6.6\%) positive for both P53 and MDM2 using the DO-7 antibody, and 25 cases $(5.3 \%)$ when using the PAb1081 antibody. Further details with respect to the histopathologic characteristics and their relationship with P53 and MDM2 expression may be found in our previous work [2].

The mean percentage of $\mathrm{Ki}-67$ positive cells was $0.6 \%$ (range 0 to $33 \% \mathrm{SD} 2.6$ ). P53-positive cases showed significantly higher $\mathrm{Ki}-67$ expression $(0.24$ vs. $2.69 \%, \mathrm{p}<<0.01$ for DO-7 and 0.34 vs. $2.46 \%$, $\mathrm{p}<<0.01$ for PAb1081), yet the values for MDM2 positive and negative cases were only slightly different (0.52 vs. $0.66 \%$, NS). Consequently, p53-positive MDM2-positive cases showed significantly higher expression (0.54 vs. $1.51 \%, \mathrm{p}<0.05$ for DO-7 and 0.44 vs. $1.97 \%, \mathrm{p}<0.02$ for PAb1081).

The results obtained when using other immunohistochemical stains are shown in Table II. Briefly, the tumors with sarcomatoid components showed lower expression of VEGF-A (1.15 vs. 0.69; p < 0.004) whereas tumors with detectable necrosis showed lower expression of VEGF-C (0.71 vs. $0.92 ; \mathrm{p}<0.005)$ but higher VEGF-D (0.28 vs. 0.39; p < 0.04). Somewhat surprisingly, expression of CA9 was lower in tumors showing necrosis (1.98 vs. 1.45 ; $\mathrm{p}<<0.001$ ). Almost all immunohistochemical results differed when considering differences in pT stage, with CA-9 proving to be the only non-significant observation
Table IV. Correlations between immunohistochemistry and tumor diameter

\section{DIAMETER}

\begin{tabular}{cc}
\hline VEGF-A & $\mathrm{r}=0.1389, \mathrm{p}=0.012$ \\
\hline VEGF-C & $\mathrm{r}=0.0803, \mathrm{p}=0.147$ \\
\hline VEGF-D & $\mathrm{r}=0.0983, \mathrm{p}=0.075$ \\
\hline GLUT1 & $\mathrm{r}=0.1106, \mathrm{p}=0.045$ \\
\hline CK7 & $\mathrm{r}=-0.1226, \mathrm{p}=0.026$ \\
\hline CA9 & $\mathrm{r}=-0.1721, \mathrm{p}=0.002$ \\
\hline
\end{tabular}

(Table III). Tumor diameter showed a weak correlation with immunohistochemical marker expression (Table IV). Almost all immunohistochemical data differed when tumor grade was also considered (evaluation by both the Fuhrman and Delahunt methods was undertaken; see Tables V and VI). However, the difference observed when quantifying GLUT1 expression was not significant, although close to significance when graded according to Fuhrman.

There was a significant difference in VEGF-A expression between P53 negative and positive cases (for DO-7 stain 1.17 vs. 0.86; for PAb1081 1.17 vs. 0.87; both $\mathrm{p}<0.001)$ as well as MDM2 negative and positive cases (1.05 vs. $1.25 ; \mathrm{p}<0.001)$. P53 negative and positive cases evaluated using the DO-7 antibody differed also in their CK 7 expression (0.14 vs. 0.23 ; $\mathrm{p}<0.05)$. When cases were evaluated using the PAb1081 antibody, P53 negative and

Table V. Relationship between the immunohistochemistry results and grading by the standard method

\begin{tabular}{ccccccccccccc}
\hline & \multicolumn{2}{c}{ VEGF-A } & \multicolumn{2}{c}{ VEGF-C } & \multicolumn{2}{c}{ VEGF-D } & \multicolumn{2}{c}{ GLUT1 } & \multicolumn{2}{c}{ CK-7 } & \multicolumn{2}{c}{ CA9 } \\
\hline GRADE & MEAN & SD & MEAN & SD & MEAN & SD & MEAN & SD & MEAN & SD & MEAN & SD \\
\hline 1 & 0.83 & 0.70 & 0.53 & 0.53 & 0.15 & 0.30 & 0.35 & 0.54 & 0.23 & 0.37 & 2.19 & 0.88 \\
\hline 2 & 1.16 & 0.76 & 0.75 & 0.68 & 0.29 & 0.46 & 0.36 & 0.49 & 0.14 & 0.32 & 1.87 & 0.97 \\
\hline 3 & 1.59 & 0.86 & 1.06 & 0.70 & 0.50 & 0.60 & 0.50 & 0.55 & 0.10 & 0.28 & 1.48 & 0.91 \\
\hline 4 & 0.78 & 0.73 & 0.77 & 0.61 & 0.35 & 0.54 & 0.49 & 0.56 & 0.15 & 0.32 & 1.83 & 1.03 \\
\hline $\mathrm{p}$ & $<<0.001$ & $<<0.001$ & $<<0.001$ & $<0.07$ (N.S.) & $<0.03$ & $<<0.001$ \\
\hline
\end{tabular}


Table VI. Relationship between the immunohistochemistry results and grading by the Delahunt [5] method

\begin{tabular}{ccccccccccccc}
\hline & \multicolumn{2}{c}{ VEGF-A } & \multicolumn{2}{c}{ VEGF-C } & \multicolumn{2}{c}{ VEGF-D } & \multicolumn{2}{c}{ GLUT1 } & \multicolumn{2}{c}{ CK-7 } & \multicolumn{2}{c}{ CA9 } \\
\hline GRADE & MEAN & SD & MEAN & SD & MEAN & SD & MEAN & SD & MEAN & SD & MEAN & SD \\
\hline 1 & 0.98 & 0.74 & 0.63 & 0.61 & 0.22 & 0.39 & 0.37 & 0.52 & 0.19 & 0.35 & 2.06 & 0.92 \\
\hline 2 & 1.50 & 0.88 & 0.99 & 0.73 & 0.43 & 0.59 & 0.41 & 0.48 & 0.10 & 0.28 & 1.55 & 0.96 \\
\hline 3 & 1.52 & 0.84 & 1.05 & 0.66 & 0.46 & 0.58 & 0.53 & 0.58 & 0.08 & 0.23 & 1.22 & 0.83 \\
\hline 4 & 0.71 & 0.73 & 0.78 & 0.65 & 0.37 & 0.55 & 0.50 & 0.56 & 0.18 & 0.34 & 1.99 & 0.98 \\
\hline $\mathrm{p}$ & $<<0.001$ & $<<0.001$ & $<0.001$ & \multicolumn{2}{c}{$\mathrm{NS}$} & & $<0.05$ & $<<0.001$ \\
\hline
\end{tabular}

positive cases differed in VEGF-C expression $(0.73$ vs. $0.97 ; \mathrm{p}<0.01)$. The immunohistochemical parameters studied did not differ significantly between p53-positive MDM2-positive cases and the rest of the cases studied; however, $\mathrm{Ki}-67$ was an exception (see above).

\section{Discussion}

Renal cell carcinoma constitutes just $9 \%$ of human cancers [6], but is of interest to both the urologist and the urologic pathologist, due to the fact that it is the most aggressive of urologic malignancies. Furthermore, significant progress has been made of late both in understanding its biology and improving treatment. Prognostication in RCC is difficult and the best established morphologic prognostic factors include tumor type and stage, presence of sarcomatoid components, and for some subtypes, histologic grade [7]. Additional prognostic biomarkers would be highly clinically useful; however, to date, there are only limited data available; therefore further studies are needed before they might be used in clinical practice $[7,8]$.

The TP53 gene is mutated in many human cancers, and this mutation paradoxically results in P53 protein accumulation. A significant proportion of RCC cases express the P53 protein product, yet the TP53 mutation rate in RCC is low, suggesting another, poorly understood mechanism for protein accumulation [9]. The MDM2 gene product participates in the same pathway as TP53; it is the main regulator of TP53 functions, and its expression is reciprocally controlled by a P53-dependent mechanism. It has been hypothesized that RCCs expressing both P53 and MDM2 behave in a more aggressive fashion $[1,10,11]$. We decided to further explore this idea and in our previous work [2] we found a number of morphologic differences between P53/MDM2-positive and -negative CCRCC, which could influence the prognosis of this subgroup of tumors.

The basic carcinogenic mechanism in CCRCC involves abnormal activation of the so-called "hypoxia pathway". Furthermore, the most frequent cytogenetic event, a $3 \mathrm{p}$ deletion, leads to loss of the $V H L$ gene. The $V H L$ protein product is responsible for inactivating hypoxia inducible factor (HIF). Both in response to hypoxia and the loss of the VHL gene, HIF begins to fulfill its role as a transcription factor, leading to the expression of a number of genes. The protein products of these genes participate in both protecting the cell against hypoxia and activating angiogenesis. Among the genes in question there are carbonic anhydrase 9, vascular endothelial growth factors and their receptors, glucose transporter 1 and others [12-16].

Carbonic anhydrase 9 (CA9), like the other carbonic anhydrases, catalyzes the reversible hydration of carbon dioxide. This enzyme is not expressed in non-neoplastic kidney cells, and in several cancers CA9 has been shown to be a marker of hypoxia. Liao et al. [17] described the immunohistochemistry for CA9 as highly specific for CCRCC, and since then it has been used for diagnostic purposes. Genega et al. [18] studied CA9 expression in a large, heterogeneous group of renal tumors and confirmed that its expression is significantly more common in CCRCC, and in this tumor type is associated with the histologic grade. Sandlund et al. [19] analyzed the CA9 expression in different renal carcinomas, confirming its relative specificity for CCRCC. They failed to find a correlation between CA9 expression, tumor stage and grade, yet observed that tumors with low CA9 expression behave in a considerably more aggressive fashion and this effect was evident also in multivariate analysis. On the other hand, some of the most recent papers have failed to show the prognostic significance of CA9 [20, 21]. Zerati et al. [20] analyzed a group of non-metastatic RCCs, and found no relationship between CA9 and overall survival, tumor stage, size, invasiveness or vascular invasion. Zhang et al. [21] analyzed a large cohort of RCC cases with periods of observation exceeding 10 years. They found that low CA9 expression is predictive of poor prognosis in univariate analysis, both in terms of survival and time to metastasis, yet this effect disappeared when adjusted for nuclear grade and necrosis. The discordance in these results has been explained by comparing differences among the different groups of patients analyzed (i.e. entire CCRCC population vs. metastatic cases), differences in the size of the populations under 
study or which technology was used (i.e. TMAs vs. whole sections). In our study, we observed that CA9 expression was inversely associated with tumor size, stage and grade. The lower CA9 expression in the cases where necrosis was evident may be due to the fact that CA9 regulation in CCRCC is not related to hypoxia itself, but to autonomous activation of the hypoxia pathway, characteristic for this cancer. Lastly, CA9 has also been proposed as a predictive marker in CCRCC. Atkins et al. [22] analyzed the influence of CA9 expression on immunologic treatment and concluded that only patients with tumors expressing high CA9 levels could benefit with prolonged survival after treatment with interleukin-2. More recently, Choueiri et al. [23] analyzed a group of metastasizing RCCs for CA9 expression. They concluded that although CA9 is not a prognostic factor in this particular subgroup of patients, it may serve as a predictor of treatment results with anti-angiogenic drugs.

Different vascular endothelial growth factors (VEGFs) participate in the development of blood vessels and lymphatic vessels. Of these, VEGF-A is the main regulator of blood vessel growth and differentiation. In CCRCC it is thought to act, in concert with its receptor, as the main autocrine stimulating loop for cancer cells. Following the understanding of this fact, antiangiogenic treatment for advanced CCRCC was developed. Furthermore, VEGF-A and its receptor VEGFR1 are the most important of the VEGF family when considering molecular pathogenesis in RCC. Accordingly, Gunningham et al. [24] failed to show upregulation of VEGF-C mRNA in CCRCC. They also observed no increase of VEGFR2 or VEGFR3, but reported a significant correlation between levels of VEGF-C and VEGFR3. In accordance with our previous discussion above, Lakovlev et al. [25] found a positive correlation between VEGF-A expression and tumor grade, when adjusted for the expression in normal renal medullary tissue. Furthermore, they also found an inverse association between microvascular density and survival, when adjusted similarly for expression in normal renal medullary tissue. Jacobsen et al. [26] observed a correlation between VEGF-A expression and survival, but only when analyzed with a univariate model. Baldewijns et al. [27] showed that CCRCC has only limited lymphangiogenic potential, and in fact were able to show that the density of lymphatic vessels was lower in cancer tissue when compared with non-neoplastic kidney tissue. Likewise, while the VEGF-A and C mRNA level were higher in cancer samples, VEGF-D mRNA level was higher in normal tissue. Interestingly, VEGF-D expression was quite low in comparison with other VEGF types in the samples studied during our investigation. Klatte et al. constructed a molecular prognostic model that included expression of $\mathrm{Ki}-67$, VEGFR-1, VEGF-D and p53. This model showed higher power in predicting disease-free survival than standard systems, and when incorporating the standard clinicopathological data, correctly predicted the outcome in over $90 \%$ of cases [8].

Glucose transporter 1 (GLUT1) facilitates glucose transport across the cellular membrane, and is responsible for basic intracellular glucose uptake [28]. It has been shown that hypoxia induces GLUT1 expression. Abnormal HIF activation, such as the one seen in CCRCC, may cause the same effect [12]. Lidgren et al. [29] have shown that most cases of CCRCC express high levels of GLUT1. Interestingly, in contrast to papillary RCC, they found no correlation between GLUT1 expression and tumor stage. This contrasts with data obtained in the present study in that we observed a weak, yet significant, correlation between this marker and tumor diameter. Lidgren et al. observed low GLUT1 expression and determined that it was a favorable prognostic indicator both in papillary and clear cell RCC. Another interesting relationship was reported by Singer et al. [30], who found an inverse correlation between GLUT1 expression levels and effector T-lymphocyte activity. Therefore, hypoxia itself or activation of the hypoxia pathway might contribute to tumor-permitting immunosuppression.

Cytokeratin 7 (CK7) has a firmly established role as a diagnostic marker in renal tumor histopathology, especially because of its distinct staining pattern which is characteristic for chromophobe carcinoma $[13,31]$. Only a subset $(10-15 \%)$ of CCRCCs express $\mathrm{CK} 7$, and according to some reports, this expression may have an influence on prognosis. Mertz et al. [32] analyzed the expression of both CK7 and CK19. The CK7 and CK19 positive tumors tended to show fewer cytogenetic alterations and patients had a better prognosis. The significance of this observation was also noted when evaluating CK7 expression using multivariate analysis. Likewise, in our material, CK7 expression was seen in lower stage, lower grade tumors.

As a proliferative marker, $\mathrm{Ki}-67$ appears to be an obvious prognostic factor, yet several studies have failed to confirm this in multivariate models. In the study by Kramer et al., [33] Ki-67 expression was found to have an influence on survival, but this was seen only when univariate analysis was employed, whereas in multivariate models, the stage was the only variable of prognostic importance. The cited study analyzed a relatively small group of cases; and further, the material studied contained samples that were not only from clear cell carcinomas, but also other subtypes, which may have influenced the results. The results obtained by Cheville et al. [34] were more conclusive in that they analyzed a uniform group of pT1 CCRCCs and found that Ki-67 expression was significantly higher in the tumors that proved fatal, but the difference disappeared when ad- 
justed for stage, grade and presence of necrosis. In contrast, Tollefson et al. [35] specifically addressed the relationship between $\mathrm{Ki}-67$ expression and necrosis, and came to the conclusion that although a correlation between the two was present, they both contributed more strongly as independent read-outs of prognosis. The prognostic significance was also apparent when these data were evaluated using a multivariate model which took into account stage, grade and clinicopathologic parameters. Kankuri et al. [36] reported that tumors expressing both $\mathrm{P} 53$ and $\mathrm{Ki}-$ 67 showed higher frequency of metastases. They also observed, similar to our study, an association between P53 and Ki-67 expression. On the other hand, Moch et al. reported that Ki-67 expression appeared to be related to survival; however, when evaluated using a multivariate model, only the influence of P53 and stage remained significant [37].

In conclusion, we have analyzed a number of potential prognostic markers in the context of $\mathrm{P} 53$ and MDM2 double expression in renal clear cell carcinoma. Our results suggest that, with the exception of $\mathrm{Ki}-67$, there are no significant associations between the markers evaluated and P53+/MDM2+ phenotype.

\section{Acknowledgements}

The authors wish to thank Mr. Robert L. Myette, who helped with manuscript editing, and Mr. Krzysztof Skomski, who prepared the microphotographs.

\section{The authors declare no conflict of interest.}

\section{References}

1. Noon AP, Polanski R, El-Fert AY, et al. Combined p53 and MDM2 biomarker analysis shows a unique pattern of expression associated with poor prognosis in patients with renal cell carcinoma undergoing radical nephrectomy. BJU Int 2012; 109: $1250-1257$

2. Hejnold M, Dyduch G, Białas M, et al. Selected morphologic features influencing the prognosis of conventional renal cell carcinomas co-expressing P53 and MDM2. Pol J Pathol 2014; 65: 29-33.

3. Lopez-Beltran A, Scarpelli M, Montironi R, et al. 2004 WHO classification of the renal tumors of the adults. Eur Urol 2006; 49: 798-805

4. Delahunt B, Cheville JC, Martignoni G, et al. The International Society of Urological Pathology (ISUP) grading system for renal cell carcinoma and other prognostic parameters. Am J Surg Pathol 2013; 37: 1490-1504.

5. Delahunt B, McKenney JK, Lohse CM, et al. A novel grading system for clear cell renal cell carcinoma incorporating tumor necrosis. Am J Surg Pathol 2013; 37: 311-322.

6. Jemal A, Bray F, Center MM, et al. Global cancer statistics. CA Cancer J Clin 2011; 61: 69-90.

7. Flanigan RC, Polcari AJ, Hugen CM. Prognostic variables and nomograms for renal cell carcinoma. Int J Urol 2011; 18: $20-31$
8. Klatte T, Seligson DB, LaRochelle J, et al. Molecular signatures of localized clear cell renal cell carcinoma to predict disease-free survival after nephrectomy. Cancer Epidemiol Biomarkers Prev 2009; 18: 894-900.

9. Imai Y, Strohmeyer TG, Fleischhacker M, et al. P53 mutations and $\mathrm{mdm}-2$ amplification in renal-cell cancers. Mod Pathol 1994; 7: 766-770

10. Noon AP, Warburton HE, Shawki H, et al. MDM2 and p53 coexpression is associated with poor prognosis in renal cell carcinoma patients undergoing radical nephrectomy. BJU Int 2008; 101: 2-2.

11. Warburton HE, Brady M, Vlatkovic N, et al. p53 regulation and function in renal cell carcinoma. Cancer Res 2005; 65: 6498-6503.

12. Chen C, Pore N, Behrooz A, et al. Regulation of glut 1 mRNA by hypoxia-inducible factor- 1 . Interaction between $\mathrm{H}$-ras and hypoxia. J Biol Chem 2001; 276: 9519-9525.

13. Okon K. Pathology of renal tumors in adults. molecular biology, histopathological diagnosis and prognosis. Pol J Pathol 2008; 59: 129-176.

14. Medina Villaamil V, Aparicio Gallego G, Santamarina Cainzos $\mathrm{I}$, et al. Searching for Hif1-alpha interacting proteins in renal cell carcinoma. Clin Transl Oncol 2012; 14: 698-708.

15. Clifford SC, Prowse AH, Affara NA, et al. Inactivation of the von Hippel-Lindau (VHL) tumour suppressor gene and allelic losses at chromosome arm $3 p$ in primary renal cell carcinoma: evidence for a VHL-independent pathway in clear cell renal tumourigenesis. Genes Chromosomes Cancer 1998; 22: 200-209.

16. Pantuck AJ, Zeng G, Belldegrun AS, et al. Pathobiology, prognosis, and targeted therapy for renal cell carcinoma: Exploiting the hypoxia-induced pathway. Clin Cancer Res 2003; 9: 46414652.

17. Liao SY, Aurelio ON, Jan K, et al. Identification of the MN/ CA9 protein as a reliable diagnostic biomarker of clear cell carcinoma of the kidney. Cancer Res 1997; 57: 2827-2831.

18. Genega EM, Ghebremichael M, Najarian R, et al. Carbonic anhydrase IX expression in renal neoplasms correlation with tumor type and grade. Am J Clin Pathol 2010; 134: 873-879.

19. Sandlund J, Oosterwijk E, Grankvist K, et al. Prognostic impact of carbonic anhydrase IX expression in human renal cell carcinoma. BJU Int 2007; 100: 556-560.

20. Zerati M, Leite KR, Pontes-Junior J, et al. Carbonic Anhydrase IX is not a predictor of outcomes in non-metastatic clear cell renal cell carcinoma - a digital analysis of tissue microarray. Int Braz J Urol 2013; 39: 484-492.

21. Zhang BY, Thompson RH, Lohse CM, et al. Carbonic anhydrase IX (CAIX) is not an independent predictor of outcome in patients with clear cell renal cell carcinoma (ccRCC) after longterm follow-up. BJU Int 2013; 111: 1046-1053.

22. Atkins M, Regan M, McDermott D, et al. Carbonic anhydrase IX expression predicts outcome of interleukin 2 therapy for renal cancer. Clin Cancer Res 2005; 11: 3714-3721.

23. Choueiri TK, Regan MM, Rosenberg JE, et al. Carbonic anhydrase IX and pathological features as predictors of outcome in patients with metastatic clear-cell renal cell carcinoma receiving vascular endothelial growth factor-targeted therapy. BJU Int 2010; 106: 772-778.

24. Gunningham SP, Currie MJ, Han C, et al. Vascular endothelial growth factor-B and vascular endothelial growth factor-C expression in renal cell carcinomas: Regulation by the von Hippel-Lindau gene and hypoxia. Cancer Res 2001; 61: 32063211.

25. Iakovlev VV, Gabril M, Dubinski W, et al. Microvascular density as an independent predictor of clinical outcome in renal cell carcinoma: an automated image analysis study. Lab Invest 2012; 92: 46-56 
26. Jacobsen J, Grankvist K, Rasmuson T, et al. Expression of vascular endothelial growth factor protein in human renal cell carcinoma. BJU Int 2004; 93: 297-302.

27. Baldewijns MM, Roskams T, Ballet V, et al. A low frequency of lymph node metastasis in clear-cell renal cell carcinoma is related to low lymphangiogenic activity. BJU Int 2009; 103: 1626-1631.

28. Olson AL, Pessin JE. Structure, function, and regulation of the mammalian facilitative glucose transporter gene family. Annu Rev Nutr 1996; 16: 235-256.

29. Lidgren A, Bergh A, Grankvist K, et al. Glucose transporter-1 expression in renal cell carcinoma and its correlation with hypoxia inducible factor-1 alpha. BJU Int 2008; 101: 480-484.

30. Singer K, Kastenberger M, Gottfried E, et al. Warburg phenotype in renal cell carcinoma: high expression of glucose-transporter 1 (GLUT-1) correlates with low CD8(+) T-cell infiltration in the tumor. Int J Cancer 2011; 128: 2085-2095.

31. Mathers ME, Pollock AM, Marsh C, et al. Cytokeratin 7: a useful adjunct in the diagnosis of chromophobe renal cell carcinoma. Histopathology 2002; 40: 563-567.

32. Mertz KD, Demichelis F, Sboner A, et al. Association of cytokeratin 7 and 19 expression with genomic stability and favorable prognosis in clear cell renal cell cancer. Int J Cancer 2008; 123: 569-576.

33. Kramer BA, Gao X, Davis M, et al. Prognostic significance of ploidy, MIB-1 proliferation marker, and $\mathrm{p} 53$ in renal cell carcinoma. J Am Coll Surg 2005; 201: 565-570.

34. Cheville JC, Zincke H, Lohse CM, et al. pT1 clear cell renal cell carcinoma: a study of the association between MIB-1 proliferative activity and pathologic features and cancer specific survival. Cancer 2002; 94: 2180-2184.

35. Tollefson MK, Thompson RH, Sheinin Y, et al. Ki-67 and coagulative tumor necrosis are independent predictors of poor outcome for patients with clear cell renal cell carcinoma and not surrogates for each other. Cancer 2007; 110: 783-790.

36. Kankuri M, Soderstrom K-O, Pelliniemi T-T, et al. The association of immunoreactive $\mathrm{p} 53$ and $\mathrm{Ki}-67$ with T-stage, grade, occurrence of metastases and survival in renal cell carcinoma. Anticancer Res 2006; 26: 3825-3833.

37. Moch H, Sauter G, Gasser TC, et al. p53 protein expression but not $\mathrm{mdm}-2$ protein expression is associated with rapid tumor cell proliferation and prognosis in renal cell carcinoma. Urol Res 1997; 25: S25-S30.

\section{Address for correspondence}

\section{Krzysztof Okoń}

Department of Pathomorphology

Jagiellonian University Medical College

Grzegórzecka 16

31-531 Krakow, Poland

e-mail:k.okon@cm-uj.krakow.pl 